\title{
A Critical Review of the Articles "Rubella Outbreak Investigation, Gokwe North District, Midlands Province, Zimbabwe 2014
}

\author{
Article by D. Chirundu ${ }^{1,}$ P.N. Magande ${ }^{2,}$ A.N. Musiya ${ }^{3}$ \\ ${ }_{1,2,4}$ Kadoma City Health Department, Zimbabwe \\ ${ }^{3}$ University of Zimbabwe Dept. of Community Medicine, Zimbabwe \\ E-mail:dchirundu@me.com ${ }^{1,}$ pmagande@gmail.com ${ }^{2}$,drnyashaadrianmusiya@gmail.com ${ }^{3}$, \\ skhaemoyo@gmail.com ${ }^{4}$
}

Source: This review looks at the manuscript entitled "Rubella Outbreak Investigation, Gokwe North District, Midlands Province, Zimbabwe 2014- a case control study " written by Donewell Bangure et. al. It was published in The Pan African Medical Journal Issue of September 2015. The Doi is 10.11604/pamj.2015.22.60.5939.

\begin{abstract}
This was an appraisal of a Rubella outbreak investigation carried out in Gokwe North District (Zimbabwe). The outbreak occurred from 24 ${ }^{\text {th }}$ June to 12 August 2014 at a primary school. The manuscript has an abstract that provided the stance and thesis. The introductory part covered the epidemiology of the disease. Methods section detailed the study design. Findings of the descriptive and analytical epidemiology investigations are presented in the results sections.

The article was published in the Pan African Medical Journal, a peer reviewed journal. This confers credence to the journal. It is tracked by HINARI, and Scopus, among others. The corresponding author is Donewell Bangure, a Zimbabwean epidemiologist. The manuscript was backed up by credible references from World Health Organization and other peer reviewed journals. However, wrong construction of the epidemic curve, may have led to erroneous conclusions.

Most of the information in the manuscript was objectively developed. However, some references were unpublished, and, some outdated. The manuscript was published in a peer reviewed journal and this makes it stable. There are three tables and one graph in the article. There are three well labelled tables and a graph in the manuscript. Information in the tables and graph is presented in a way that is comprehensible.
\end{abstract}

Keywords: Rubella, Gokwe North, Outbreak.

\section{Introduction}

This was an appraisal of a Rubella outbreak investigation carried out in Gokwe North District (Midlands Province) of Zimbabwe. In this review we will initially look at the literature review pertaining to the rubella outbreak investigation. We will then look at the article structure in terms of content and alignment. Under the article critique, we will give an overview of the authority, accuracy, currency, relevance, objectivity, stability and analysis of graphs or tables.

\section{Review of literature}

Rubella, is a mild febrile disease with a diffuse punctuate and maculopapular rash sometimes resembling that of measles (Heymann 2014 and Dimech et. al. 2015). It usually occurs in winter and spring. It is caused by the rubella virus, which can be found in the throat, blood, and stool of infected person. Transmission is by contact with nasopharyngeal secretions of infected people through droplet spread. Infected persons who exhibit no signs and symptoms may transmit rubella. According to the American Public Health Association (2014) 30\% to 50\% of rubella infections are not recognized as rubella disease. An increase in rubella incidence has been noted in unvaccinated adolescents and adults as well as in religious communities with no or low levels of vaccination. 
DOI: $10.21522 / \mathrm{TIJPH} .2013 .06 .02 . A r t 006$

ISSN: $2520-3134$

The incubation period, varies from 14-17 days, with a range of 14-21 days (Heyman 2014). Signs and symptoms of rubella includes fever, swollen glands that are usually found either in the neck or behind the ears, and, a rash that appears as either pink or light red spots. Other symptoms of rubella may include conjunctivitis and coryza. When rubella occurs in a pregnant woman, it may cause congenital rubella syndrome, resulting in malformations of the developing foetus (Axton et. al 1979; Kimberlin et. al 2015 and Chimhuya et. al. 2015). In areas where there is no routine rubella immunization like Cameroon and Central African Republic among others, rubella outbreaks are often confused and responded to as if they are measles outbreaks. (Chimhuya et. al. 2015; Axton et al.1979; Farra et. al.2016: and Mengouo et. al. 2017) This leads to waste of resources and provision of a false sense of security to the communities

\section{Article summary}

The article "Rubella Outbreak Investigation Gokwe North District Midlands province Zimbabwe 2014" by Bangure et. al. is based on a Rubella outbreak investigation. The outbreak occurred from $24^{\text {th }}$ of June to the $12^{\text {th }}$ of August 2014 at a primary school. The index case was a child of a relief teacher who had recently joined the school. The outbreak spread among the school pupils and ultimately infecting community members. The total number of people infected was 374 over three months. A case control study was done to identify factors associated with contracting the infection. Having a household contact, classmate contact, having more than three children in a household were significant risk factors ( $>0.05)$. Having suffered from rubella illness, and history of measles vaccination were protective but not statistically significant. Based on the shape of the epidemic curve the authors concluded that it was a propagated outbreak that spread from the school to the community. The recommendation was that a vaccine for rubella be routinely administered in Zimbabwe.

\section{Article structure}

This manuscript has an abstract that provided the stance and thesis as well as a brief overview of the main points. The article is an IMRAD outline of the outbreak investigation. Thus, there is an introduction, methods, results, discussion, and conclusion in that order.

The introduction gave a detailed background of rubella as well as the epidemiology of the disease. It also gave the details of the increase in the number of cases during the period and the index case. However, it is our opinion that the details of the index case were obtained as part of the investigation and as such it was more appropriate to be placed in results section.

Methods sections specified the study design. The definition of a case and the control as the terms applied to the investigation were clearly defined in this section. It is stated that simple random sampling was used, however, there is no elaboration on how the sample size was calculated.

Findings of the descriptive and analytical epidemiology components of the investigation are presented in the results sections. In descriptive epidemiology, "person characteristics" are presented in the demographic data. The epidemic timeline (time) is depicted by use of an epidemic curve. However, the "place attributes" are not well covered. Under the circumstances, we expected the attack rates by class, and/or village to depict the spatial distribution of cases. In analytical epidemiology, univariate, bivariate and multiple logistic regression analysis are soundly presented.

Under the discussion section, the statement "the epi-curve has several peaks typical of a propagated outbreak, suggestive of person to person transmission" is more of a conclusion than discussion. There is also inappropriate use of words like "quarantine" as the sick are isolated and those exposed and not exhibiting symptoms are quarantined (source). In the article sick pupils were said to have been "quarantined".

The conclusion was that primary school children aged 5-9 years were mostly affected. Nonetheless, there is nowhere in the results were attack rates by age are presented so that we get the basis of the conclusion. However, the recommendation to implement routine rubella vaccination is a sound recommendation.

The sentences were short, the readability was high, and, this makes the article easy to follow. 


\section{Article critique}

\section{Authority}

The journal in which the article is published Pan African Medical Journal (ISSN 1937-8688 (www.panaftrican-med-journal.com) is a peer reviewed journal that is published in partnership with the African Field Epidemiology Network (AFENET). This confers credence to the journal. It is tracked by HINARI, Scopus, DOAJ, Embase and PubMed Central. The numbers of manuscripts submitted to the journal an increased from 211 in 2010 to 2614 in 2017.Out of the 2614 manuscripts submitted in 2017, 994 were published. Manuscripts are submitted by authors from all geographical regions. This attests to the popularity of the Pan African Medical Journal and this gives it authority. Its affiliation to Field Epidemiology Training programs in Africa gives it further credence as this makes it a resource of first choice for African disease control specialists.

The corresponding author of the article is Donewell Bangure. He is a Zimbabwean public health expert formerly employed as an assistant field coordinator for the Zimbabwe field Epidemiology Training program but currently employed as an epidemiologist by CDC Africa. According to ResearchGate, he has published several articles on outbreak investigations, evaluation of surveillance systems, and, program evaluation. He is the holder of a master's in public health degree from the Zimbabwe Field Epidemiology Training Program.

\section{Accuracy}

The primary source of data for the manuscript was an outbreak investigation. It was backed up by references from credible sources like WHO (World Health Organization 2012). However, wrong construction of the epidemic curve, leading to misinterpretation of same may have led to inaccurate conclusions. The interval between the dates on the $x$-axis is supposed to be between a quarter to a third of the incubation period (Centres for Disease Control 2012).

\section{Currency}

The manuscript was accepted for publication on 26 May 2015 and published on 22 September 2015. This makes it relatively current work. The field work was done from June to August 2014. One compromise is that most the references were outdated- for example the article by Lawn et. al. was published in the year 2000. Some of the references are not published in peer reviewed journals for example the 2005 Rubella outbreak investigation by Mpeta et. al. and the 2004 outbreak by Muchedzi et. al.

\section{Relevance}

The Pan African Journal of Medicine is a journal of the African Field Epidemiology Training Programs. It is relevant in that it addresses relevant and pertinent issues of public health interest including communicable disease control in Africa and beyond.

\section{Objectivity}

To some extent, the information was objectively developed. Bias may hay have been introduced in the article by incorrect construction and interpretation of the epidemic curve. Terms are well explained and described. The sample size was mentioned but the assumptions and how it was calculated are not articulated.

\section{Stability}

The manuscript was published in a peer reviewed journal and this makes it stable. 
DOI: $10.21522 /$ TIJPH.2013.06.02.Art006

ISSN: $2520-3134$

\section{Analysis of graphs and tables}

There are three tables and one graph in the article. All tables are well labelled, and information is presented in a way that is easy to understand. However, in table 2 bivariate analysis, odds ratio as well as 95\% confidences intervals are included whilst $p$-values are not absent.

Figure 1 is an epidemic curve depicting the epidemic timeline. The date of onset is shown on the $x$-axis and the number of case on the $y$-axis. The interval on the $\mathrm{x}$-axis is one day. This is supposed to be a quarter to a third of the incubation period (WHO 2008 and CDC 2018). This defies standard epi-curve construction and makes correct interpretation of epi-curves impossible. Wrong interpretation in turn leads to incorrect conclusion on transmission dynamics.

There was need to present a spot map to depict the "place" of descriptive epidemiology.

\section{Recent advances related to the topic}

Bosma et. al. (1995) investigated the use of PCR for detection of virus RNA in clinical samples. Rubella virus RNA was detected in 12 pharyngeal swabs from patients with serologically confirmed rubella; these RT-PCR results were in complete agreement with virus isolation. Analysis of products of conception obtained after confirmed primary maternal rubella infection by RT-PCR gave $92 \%$ agreement (12 of 13 samples) with virus isolation. No false-positive results were obtained. PCR can therefore be used to establish the source of infection. Molecular investigations of Rubella is also being done in countries like Romania (Lazar et. al. 2016) In the case of Bangure et. al. they could have compared the PCR result from Gokwe and Masvingo to verify if the outbreaks were related (Bosma et. al).

Laboratory tests are essential for confirming sporadic cases and outbreak of rubella. Detection of rubella is often necessary to confirm rubella cases and to identify specimens to be used to characterize wild-type rubella viruses. Zhen Zhu as far back as 2007 showed that direct RT-PCR followed by hybridization was the most sensitive method; the replicon-based method was the least difficult to perform. Such methods can be used in confirming the cases where resources allow. All the recent advances show that molecular assays are important tools in the early diagnosis of rubella and congenital rubella syndrome. In the provision of molecular epidemiological information for tracking transmission pathways and in adding to the knowledge of rubella strain distribution worldwide. Another of African countries are also introducing Rubella vaccination. This is having a further effect of strengthening meascles vaccination (Wesolowski et. al. 2016).

\section{Conclusion}

This article summarized, reviewed and critiqued Donewell Bangure`s manuscript "Rubella Outbreak Investigation Gokwe North District Midlands province Zimbabwe 2014”. The content, structures and limitations of the structure were analyzed. This contributed to literature and knowledge in that it is a documentation an outbreak investigation and audit of the response. Outbreaks are common in Africa and this manuscript can serve as a reference to other investigators.

\section{Acknowledgements}

We acknowledge the contribution of Prince Matambo and Patrick Makovere for typesetting this manuscript.

\section{References}

[1]. Axton et. al. (1979). Simultaneous Rubella and Measles Epidemics in an African Community. The Central African Journal of Medicine.

[2]. Bosma et. al. (1995) PCR for detection of rubella virus RNA in clinical samples. J. Clin. Microbiol. May 1995 vol. 33 no. 5 1075-1079.

[3]. Centres for Disease Control (2006). Principles of Epidemiology in Public Health Practice-US Department of Health and Human Services Updated May 2012. 
[4]. Chimhuya et. al. (2015). Trends of rubella incidence during a 5-year period of case-based surveillance in Zimbabwe. BMC Public Health.

[5]. Dimech et.al. (2016). Standardization of assays that detect anti-rubella virus IgG antibodies. Clin Microbiol Rev 29:163-174. doi:10.1128/CMR.00045-15.

[6]. Farra et. al. (2016). Epidemiology of primary rubella infection in the Central African Republic: data from measles surveillance 2007-2014 BMC Infectious Diseases.

[7]. Heymann, D. (2014). Control of Communicable Diseases Manual. American Public Health Association.

[8]. Kimberlin et. al. (2015) The Red Book- American Academy of Pediatrics 2015.

[9]. Lawn et. al (2000). Unseen blindness, unheard deafness and unrecorded death and disability: congenital rubella in Kumusi Ghana American J public Health.

[10]. Mengouo et. al. (2017) Epidemiology of Rubella Infection in Cameroon: a 7 -year experience of measles and rubella case-based surveillance 2008-2014. BMJ.

[11]. Mpeta T, Ndlovu et. al. Rubella Outbreak in Nkakezi, Insiza District, Matebeleland South Province (Unpublished). 2005. Google Scholar).

[12]. Lazar et. al. (2016) Epidemiological and molecular investigation of a rubella outbreak, Romania, 2011 to 2012. Euro Surveill. 2016; 21(38):pii=30345. DOI: http://dx.doi.org/10.2807/1560-7917.ES.2016.21.38.30345.

[13]. Wesolowski A et al. 2016 Introduction of rubella-containing-vaccine to Madagascar: implications for roll-out and local elimination. J. R. Soc. Interface 13: 20151101.http://dx.doi.org/10.1098/rsif.2015.1101.

[14]. World Health Organization (2008) Foodborne disease outbreaks: guidelines for investigation and control. World Health Organization.

[15]. World Health Organization (2012). Global measles and rubella strategic plan: 2012-2020. W.H.O.

[16]. World Health Organization (2012). Recommended surveillance standard of rubella and congenital rubella syndrome. World Health Organization.

[17]. Zhen Zhu (2007). Comparison of Four methods using throat Swabs to Confirm Rubella Virus Infection. J. Clin. Microbiology. 